\title{
A test of the consolidation theory with ECT patients'
}

George A. Talland

HARVARD UNIVERSITY MEDICAL SCHOOL

\begin{abstract}
Abstraet
Patients who learned a story and a map design the night before, recalled less after ECT than those who learned the same material from 5 to $90 \mathrm{~min}$. prior to ECT, or control Ss tested after a night but without ECT. Memory tended to be most intact with the shorter intervals between learning and shock. The results do not fit the consolidation theory, but can be reconciled with other findings that support it. New learning, if it involves organization, exceeds the duration of the consolidation process, and beyond that limit interference with recall increases with time.
\end{abstract}

\section{Problem}

Next to traumatic amnesias, the effects of electroconvulsive therapy (ECT) or its experimental versions have furnished the most compelling evidence for a consolidation theory of memory. The theory implies that information becomes available for subsequent recall-is learned-only if certain neural processes perseverate or reverberate after the cessation of the event that had first triggered them. Prior to consolidation, impressions are labile and vulnerable to obliteration by interfering processes.

Retroactive amnesia following concussion typically extends over periods of a few seconds or several minutes prior to the head injury (Russell \& Nathan, 1946). Though his behavior during that period appeared to be normal, the patient has no recollection of the events to which he responded quite appropriately. It is inferred that this happens because the accident interrupted brain processes that are necessary for consolidating mernories. Several animal experiments tend to support the consolidation theory (cf., Glickman, 1961; Deutsch, 1962), as do some studies with ECT patients (Zubin \& Barrera, 1941; Mayer Gross, 1943). Since none of them allow for an unambiguous conclusion, another attempt was made in the present study to test the consolidation hypothesis.

\footnotetext{
Method

Ss were hospitalized patients who were given ECT twice or three times weekly. Shock lasted 3 to $5 \mathrm{sec}$., and followed administration of a muscle relaxant and anesthetic.

Memory and related functions were tested by one or two tasks as closely before anesthesia as was practicable, and again about $1 / 2$ hr. after the patient had regained consciousness. The experimental group consisted of eight patients who received ECT early in the morning and were therefore given their pre-treatment learning tests the night before, immediately or shortly before they would retire to sleep. These patients will be referred to as the the overnight group. The principal control group consisted of 24 patients who were given ECT later in the morning and were tested at intervals ranging from 5 to $90 \mathrm{~min}$. prior to treatment; they will be referred to as the morning group. A control group of 12, drawn from the same ward as and matched for age with the overnight group, was tested on the night before and on mornings when they were not given treatment.
}
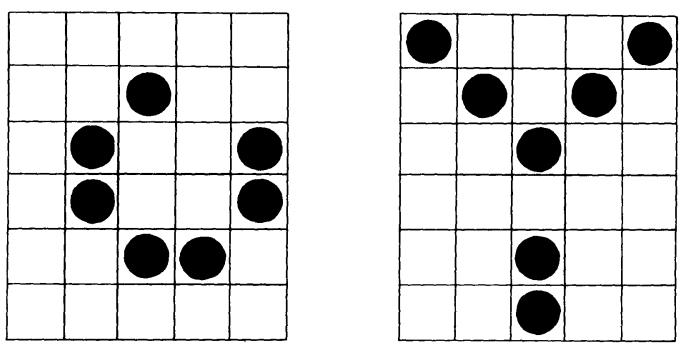

Fig. 1. Patterns presented for reconstruction.

The control group was tested once; overnight and morning groups learned the story before and after shock the second and seventh, the map before and after shock the third and sixth treatment day. After learning the story and again, following ECT, before its recall, $\mathrm{S}$ was also tested for the running digit span. Two matched stories, that have been used in previous studies (cf., Talland, 1965, pp.236-237) were presented in balanced order, readaloud once by $\mathrm{E}$ at a measured pace. Recall was scored on three criteria: (a) remembering that a story had been read; (b) recall of three principal units of content; (c) reproduction of 16 detailed content units, in the original phrasing or in suitable alternate words.

The maps were simple patterns constructed with seven discs on a $5 \times 6$ checkerboard, as illustrated in Fig. 1. Their critical feature was the position of the gap. The pattern was displayed for $60 \mathrm{sec}$, or-if S so desired-for $90 \mathrm{sec}$. Then the checkers were swept to the side and $\mathrm{S}$ was asked to reconstruct it. $\mathrm{E}$ advised him whether his attempt was successful, and recorded the number of trials-up to six-required for correct performance. There was no time limit and, upon successful reconstruction, $\mathrm{S}$ was advised to remember the design as he would be asked to reproduce it later. Following ECT the reconstruction test was identical with the first but, if $\mathrm{S}$ failed, it was followed by a recognition test. For this the pattern was shown without a gap, and $S$ was asked to identify it. If he reported it as different from the original design, he was asked to point out the difference. Recognition, if incorrect, was followed by a test of relearning modelled on the original procedure of reconstruction.

\section{Results}

Table 1 compares the three groups on three measures of forgetting. Following immediately after its first (pre-treatment) presentation every $\mathrm{S}$ had remembered hearing a story, but one member of each ECT group and four control Ss had retained none of its content. After shock the former two could not remember learning a story, butall four control Ss recalled it in the morning. Judged by reproduction of content and details, the three groups did not differ significantly in the first learning test; neither did the overnight and morning groups differ significantly in the running memory span before or after ECT。

One morning and two overnight patients failed to reconstruct the map in six trials on first learning, but were successful with the parallel task in the second session; three control Ss failed on this test. Excluding these instances, reconstruction after ECT was correct as shown in Table 2. The three successes on first trial in the overnight group represent as many different Ss, 
Table 1. Delayed recall of story: $\mathrm{N}$ of Ss who forgot hearing story, retained none of its principal contents, or less than 5 details; classified according to interval between learning and treatment.

\begin{tabular}{|c|c|c|c|c|c|c|c|c|}
\hline & \multicolumn{2}{|c|}{$\mathrm{N}$} & \multicolumn{2}{|c|}{ No recall of story } & \multicolumn{2}{|c|}{ No recall of content } & \multicolumn{2}{|c|}{$\begin{array}{l}\text { Recall of less } \\
\text { than } 5 \text { units }\end{array}$} \\
\hline & \multicolumn{2}{|c|}{ Session } & \multicolumn{2}{|c|}{ Session } & \multicolumn{2}{|c|}{ Session } & \multicolumn{2}{|c|}{ Session } \\
\hline & 1 & 2 & 1 & 2 & 1 & 2 & 1 & 2 \\
\hline $\begin{array}{l}\text { Control group } \\
\text { (overnight; no ECT) }\end{array}$ & 12 & - & 0 & - & 3 & - & 6 & - \\
\hline Overnight group & & & & & & & & \\
\hline over 10 hours & 11 & 11 & 6 & 7 & 8 & 8 & 8 & 8 \\
\hline Morning group* & 22 & 21 & 3 & 1 & 11 & 6 & 11 & 10 \\
\hline $61-90 \mathrm{~min}$. & 4 & 5 & 0 & 0 & 1 & 3 & 1 & 3 \\
\hline $31-60 \mathrm{~min}$. & 8 & 6 & 2 & 0 & 5 & 1 & 5 & 3 \\
\hline 21-30 $\mathrm{min}$ & 1 & 3 & 0 & 0 & 1 & 1 & 1 & 1 \\
\hline $11-20 \mathrm{~min}$. & 6 & 5 & 1 & 0 & 1 & 0 & 1 & 2 \\
\hline $5-10 \mathrm{~min}$ & 3 & 2 & 0 & 1 & 3 & 1 & 3 & 1 \\
\hline
\end{tabular}

Note: *Interval between learning and ECT was not regularly the same for a S on 1 st and 2nd day.

the 26 instances in the morning group include repeated successes by six Ss. After failure in reproduction, two overnight patients (one in both sessions), six morning patients (none twice) and three control Ss accurately located the missing gap in the recognition test. One member of each ECT group and two control Ss were unable to relearn the map. Judged by savings in delayed recall or in relearning, neither ECT group showed any gain, while all but one of the control Ss did. Discussion

The time lag and the protection from interference afforded by a night's rest did not advance the consolidation of newly acquired information sufficiently to safeguard it from the disruption caused by ECT. Some learning may indeed have been lost overnight, although the evidence of the control group would argue against that, and such forgetting itself would hardly support the consolidation theory. The relationship observed between time available for consolidation before electroshock and retention is contrary to the hypothesis tested. It does not conflict with a theory of consolidation

Table 2. Delayed recall of Map: $\mathrm{N}$ of Ss who failed in tests of reproduction and recognition, classified according to interval between learning and treatment.

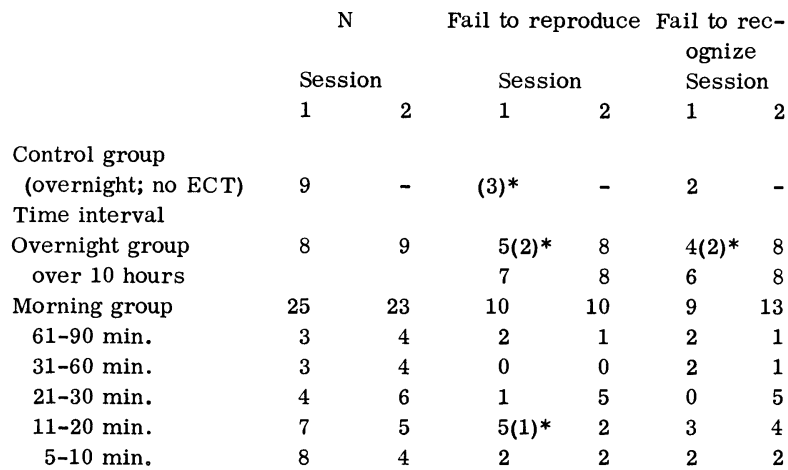

Note: Interval between learning and ECT was not regularly the same for a $\mathrm{S}$ on first and second day.

*Ss who could not learn to reproduce the map on first learning. that restricts that process to a period of less than 5 min., e.g. Cronholm \& Lagergren's (1959) conclusions based on intervals of 5 to $60 \mathrm{sec}$. between learning a single number and ECT. Moreover, the critical interval is likely to vary with the type of information to be remembered, and may depend on the processing needed to embed its content in the established categories of memory and its consequent accessibility in recall. The time range of these processes is hardly the same when the memory content is a personal experience subject to traumatic amnesias, a single number, or a story.

Consolidation is the neural process of learning or registration that demands little or no organization, e.g. rehearsing a number or fitting in an experience in the chain of expectancies and confirmations within a program. Learning a story or a map, however, involves cognitive processes that exceed the time span of consolidation. Their recall depends primarily on the recovery of an appropriate set, and this becomes more difficult as non-specific interference accumulates over time as well as after such specific interference as electric shock. Combined, these two sources of interference should indeed result in more forgetting than either does alone.

\section{References}

CRONHOLM, B., \& LAGERGREN, A. Memory disturbances after electroconvulsive therapy: III. An experimental study of retrograde amnesia after electroshock treatment. Acta Psychiat., Scand., Copenhagen, 1959, 34, 283-310.

DEUTSCH, J.A. Higher nervous function: the physiological bases of memory. Annu. Rev. Psychol., 1962, 24, 259-286.

GLICKMAN, S. E. Perseverative neural processes and consolidation of memory trace. Psychol. Bull., 1961, 58, 218-233.

MAYER GROSS, W. Retrograde amnesia. Lancet, 1943, 2, 603. RUSSELL, W. R., \& NATHAN, P.W. Traumatic amnesia. Brain, 1946, 280-300.

TALLAND, G. A. Deranged memory. A psychonomic study of the amnesic syndrome. New York: Academic Press, 1965.

ZUBIN, J., \& BARRERA, S. E. Effect of electroconvulsive therapy on memory. Proc. Soc. Exp. Biol. Med., 1941, 48, 596-597.

Note

1. This research was supported in part by the Medical Foundation of Boston and by N. I. H. research career grant MH (now HD) 15,418 . 